\title{
The Fire Chief microworld generating program: An illustration of computer-simulated microworlds as an experimental paradigm for studying complex decision-making behavior
}

\author{
MARY M. OMODEI \\ La Trobe University, Bundoora, Victoria, Australia \\ and \\ ALEXANDER J. WEARING \\ University of Melboume, Melbourne, Victoria, Australia
}

\begin{abstract}
Computer-simulated microworlds bridge the gap between the complexity of field investigations and the rigor of laboratory studies. The purpose of this paper is to draw attention to the major methodological issues involved in developing and using computer-simulated microworlds for the psychological study of complex decision-making behavior. These issues comprise flexibility and generality in the microworlds that can be created, adequate psychological validity, automatic trial administration and data logging, capacity to interface simulations of psychological processes, and adequate testing and documentation. Each of these issues is illustrated by indicating how it has been addressed in Fire Chief, a microworld generating program specifically designed for creating realistic, yet controllable, decision-making task environments in the psychology laboratory. Fire Chief (Omodei \& Wearing, 1993a) is introduced as both fully portable to other research teams and suitable for use in many different contexts requiring a complex task situation.
\end{abstract}

There has been a constant tension in psychology between research in the field and research in the laboratory. This tension raises particular problems in the study of complex decision-making behavior. It is important for psychological inquiry that the roots of this tension be addressed, if for no other reason than that many (if not most) psychologically important real-world phenomena require individuals and groups to engage in complex decision-making behaviors. Whether the focus is on quality of performance, on high-level cognitive processes, or on emotional reactions, one of two different research strategies is adopted for the study of complex decision making: laboratory studies in which precise control and measurement is achieved at the expense of external validity; and field/observational studies in which less intrusive assessment procedures result in ambiguity concerning precisely what has been measured.

Brehmer and Dörner (1993) have recently suggested that the root of this tension lies in the difficulties posed by complexity: the uncontrollable complexity of the field

The development of the Fire Chief program and the preparation of the program manual were supported by the Australian Research Council, Grant A78931183. We gratefully acknowledge the extensive programming support provided by Ben Johnston throughout all stages of Fire Chief development. Correspondence should be addressed to M. M. Omodei, School of Behavioural Health Sciences, La Trobe University, Bundoora, Victoria 3083, Australia (e-mail: m.omodei@ latrobe.edu.au). creates problems of inference, while the controlled simplicity of the laboratory may generate irrelevant findings. With respect to complexity, naturally occurring decision situations typically involve (1) a large number of interrelated variables; (2) ambiguity, intransparency, and uncertainty in the values of key variables; and (3) multiple decision alternatives.

Besides complexity, we suggest that two other characteristics of real-life decision environments are typically absent from laboratory-based investigations but are essential aspects of the experience of persons who act in these environments. First, real-life environments are usually dynamic with respect to participants' activity. What one does changes the situation itself, with subsequent activity occurring in an environment that is determined partly by previous activity. Second, real-life environments exist in real time. The environment continually changes even if no action is taken by the participant (e.g., fires spread in the absence of any firefighting activity). As a result, the when of an action is often more important than the what (a fire break burnt early in a fire situation will be more effective than one built later).

In addition to the difficulties for interpretation that field investigations pose, many psychologically important real-world phenomena cannot be studied directly. Practical, political, ethical, and/or financial constraints restrict the extent to which theoretically relevant variables can be identified, measured, and/or systematically manipulated. 


\section{Computer-Simulated Microworlds as an Emerging Experimental Paradigm}

The capabilities of present-day desktop computers offer the opportunity for a feasible compromise between field and laboratory research by allowing laboratory studies to be conducted with controlled task environments that imitate naturally occurring situations. Realistic environments that are created by computer-simulation techniques have come to be known as microworlds. This term appears to have been first used by Turkle (1984) to refer to the carefully constructed, graphically rich, and complex rule-governed worlds of video games, simulations that have much in common with the microworld environments that are now emerging for use in psychological research. This new technology partly bridges the gap between the inherent complexity of a field investigation and the rigor of a laboratory study. A microworld methodology, therefore, allows for the study of a wide range of until-now unexaminable motivational, emotional, behavioral, and physiological responses to complex and realistic decision-making situations.

In addition to the opportunities that computer-simulated microworlds provide for creating real-world analogues for the study of complex decision making, such microworlds can also be used more generally for creating realistic experimental task situations, regardless of whether decision making is the focus of research interest. That is, microworld scenarios provide a suitable experimental paradigm for any investigation requiring a controlled, complex, and dynamic task situation.

\section{Existing Computer-Simulated Microworlds: Suitability for Research Into Complex Decision Making}

The use of several types of computer-simulated microworld programs for the experimental investigation of psychological phenomena has been reported in the psychological literature. In examining the potential suitability of these computer-simulated tasks for research into complex decision-making behavior, the reader is drawn to the distinction between (1) the execution of complex perceptual-motor skills and (2) high-level decision-making activity. In the execution of perceptualmotor skills, regardless of how complicated these skills might be, the required actions are consistent and clearly specifiable. Complex decision making, on the other hand, is required precisely in those situations in which the required actions are not clear. These two types of situations require, respectively, automatic and controlled information-processing activity. As demonstrated by Schneider and Shiffrin (Schneider, Dumais, \& Shiffrin, 1984), and more recently by Ackerman (1987), the efficient execution of a complex perceptual-motor skill relies on the required actions' having become routinized, involving automatic information processing activity only. Complex decision making, on the other hand, requires the continuous application of controlled information processing. Therefore, if a computer-simulated pro- gram is to be suitable for the psychological study of naturally occurring complex decision-making processes, it is important that it require persons to engage in complex high-level decision-making activity as distinct from the execution of specific highly routinized perceptual-motor skills.

Additional criteria that must be met by a computersimulated complex decision-making microworld are that the task (1) imitate naturally occurring decision environments, (2) allow for precise experimental manipulation of characteristics of psychological interest, (3) be suitable for use with most subject populations, and (4) not require excessively long training periods.

Three general sources of computer-simulated microworld programs can be identified: computerized training simulators (e.g., flight simulators, management training games, etc.), recreational video games (e.g., fantasy adventure games, arcade games, etc.), and simulations specifically developed for theoretically based research into complex decision-making processes. Although any of these program sources might be expected at first glance to meet the previously mentioned criteria for the study of complex decision-making behavior, closer examination of the defining characteristics of computerized training simulators and recreational video games leads us to reject such simulations as a source of suitable tasks for the study of complex decision making.

Computerized training simulators are generally of two kinds: those developed for "skills acquisition" in complex environments and those developed for training in "process control" in industrial settings. Skill acquisition tasks are either rule bound, like Kanfer and Ackerman's (1989a, 1989b) air-traffic controller task, in which appropriate skill acquisition resides in learning the correct application of the rules rather than in making choices between action alternatives, or perceptually motor driven, like Decoy (Omodei, 1990) and the Space Fortress game (Mané \& Donchin, 1989), in which appropriate skill acquisition resides in developing the ability to make fine perceptual discriminations and to execute efficient motor responses automatically (Donchin, 1989). Neither type of skill acquisition task is suitable for the study of complex decision-making behavior, precisely because they both involve primarily automatic, as distinct from controlled, information-processing activity.

Process control simulations are simulations of automated industrial processes, processes that are typically controlled by human operators at consoles some distance from the plant itself(e.g., Morris \& Rouse's, 1985, Plant task). Such process control tasks are specifically targeted to impart a specific learning experience or to instruct a particular population in a specific skill or set of skills. There are several implications of such specificity for the suitability of process control tasks for the study of complex decision-making behavior. First, participants are assumed to have to some extent a specialist's knowledge of the relevant system variables and system dynamics-a knowledge that is not likely to be held by persons who are available as research subjects. Sec- 
ond, the operational conditions and system parameters of training simulations cannot be manipulated with the degree of precision and flexibility required for rigorous experimental investigation.

Recreational video games also represent a source of computer-simulated microworlds. Whereas training simulators were developed to train persons to deal with specific areas of reality, recreational video games were designed to maximize individual "enjoyment" (or, more cynically, to motivate persons to voluntarily invest money in these games). Unfortunately, the structure of recreational video games is such that these simulations cannot be readily adapted for the precise experimental study of complex decision-making behavior. First, fidelity to some aspect of reality is rarely present, the aim of the game being to place the participant in a fantasy world. Second, a limitation of commercially developed software systems is that they do not provide the user with the facility to modify system parameters, nor do they provide information on the underlying system structure (whether these be rule sets or mathematical models). Third, to be commercially successful, video games are usually so complex that many hours of play are required to achieve mastery of the task, making it impractical in terms of both financial and human resources to adopt such tasks for large-sample psychological research.

Reports of the development, and subsequent use, of microworld simulations specifically for theoretically based research into complex decision-making processes are still sparse. This can be attributed partly to the lack of adequate computing facilities for their development and use, and partly to the expense and time involved in the development of such microworlds. Almost all this research has been produced by Dörner and associates (e.g., Dörner, Kreuzig, Reither, \& Stäudel, 1983; Dörner, Stäudel, Strohschneider, 1988) and by Brehmer and associates (e.g., Brehmer, 1992; Brehmer \& Allard, 1991). Unfortunately, most of the programs that have been developed for this research suffer from the lack of a realtime component. That is, as indicated in the introductory comments to this paper, most real-life environments continually change regardless of any decision input from human participants. For the same reasons as those identified above-namely, the lack of adequate computing facilities and the expense and time involved-the development of real-time microworld simulations is of even more recent origin, and research with these microworlds is more limited in scope. To date, the only published findings of research into complex decision making using realtime microworlds are those reported by Brehmer and associates using Dessy, a firefighting simulation (Brehmer \& Allard, 1991) and NewFire, a PC-based firefighting simulation that shares many features with the Dessy simulation (Brehmer \& Løvborg, 1991). Dörner and associates have also developed several real-time microworld simulations, but their research findings remain unpublished as yet. These microworlds include Manutex (a textile factory), Maschine (a chemical production plant), and Feuer (a forest firefighting simulation).

\section{General Principles for the Efficient Development and Use of Computer-Simulated Microworlds}

Despite the present availability of relatively inexpensive computing power for supporting the use of computersimulated microworlds, the costs of software development, testing, and documentation remain high, particularly in relation to characteristics of the user interface. Furthermore, running experiments with such tools is also costly: subjects must often be trained individually in the use of the microworld, supervised carefully over a sufficient length of time to achieve an adequate level of task mastery, and then administered lengthy or numerous trials to produce stable performance data.

Consequently, investigators who embark on the task of developing microworld simulations should develop simulations that are portable, flexible, and easy to use. Such portable simulations should be suitable for use by independent research groups and be capable of addressing a diversity of research questions in various populations, by means of a variety of methodological techniques. In addition to reducing the overall costs of using computersimulated microworld methodologies, simulation portability and flexibility will allow for investigations of the validity and generalizability of any findings from microworld experiments.

\section{Development of the Fire Chief Microworld Generator}

Fire Chief (Omodei \& Wearing, 1993a) was developed to be a general-purpose program for creating realistic complex laboratory task environments in general and for creating complex decision-making environments in particular. Forest firefighting was selected as the default microworld because it was judged to be suitably complex, realistic, inherently interesting, and readily understood by subjects. Nevertheless, particular attention has been paid in developing Fire Chief to allow for the creation of many different microworlds, not necessarily those similar to fires and firefighting. The main features implemented in our first version of the program took as their starting point a small $(30 \mathrm{~K})$ freeware computer game, FIRE (Sprinsock, 1985) and the preliminary versions of Brehmer's Dessy and Dörner's Feuer.

\section{Forest Firefighting: The Default Fire Chief Microworld}

\section{Descriptions of Fire Chief Program Features in Terms of a Firefighting Microworld}

To provide a concrete context within which to present the structure and functioning of Fire Chief, the program is described in terms of a specific implementation: namely, the default firefighting microworld. Nevertheless, in customizing Fire Chief for alternative microworlds, other meanings (as cover stories) can be readily ascribed to the various program data structures, routines, and user-modifiable parameters in place of their counterparts in the firefighting microworld. For example, the variables that represent wind conditions in the 
simulated forest fires could equally appropriately be used to represent ocean currents in, say, the simulation of an oil spill at sea.

\section{The Firefighting Microworld as Experienced by Subjects: Generic Instructions}

Fire Chief is of necessity a complex program, in relation both to the demands that it may potentially place on participant subjects and to the large number of flexible features that it offers to researchers. Consequently, to assist the reader in understanding the program, we present the following generic version of the instructions that we typically give to subjects. Naturally the instructions will vary according to the specific parameter values and program options that have been selected. The instructions are typically accompanied by the experimenter's running a demonstration trial and drawing subjects' attention to the relevant on-screen activity (as in Figure 1).

You are to imagine that you are the Chief Fire Officer responsible for a large area on a hot, windy, summer day. The area contains forest, stock pasture, clearings, small residential areas, and water filled dams. You are situated at a command post some distance from the forest area and are seated in front of a video screen. On this screen are displayed the current fire situation in the forest, the prevailing wind conditions, any forecast change in these wind conditions, and the location and activity of the firefighting trucks and helicopters which are at your disposal.

If a segment of the landscape area is burning, fire will be shown in the particular segment, the height of the fire indicating the intensity of the fire in that particular area (see Figure 2). Once all the combustible fuel in a landscape segment is consumed by the fire, the area is considered to be destroyed and the landscape will be shown to be burnt. If you extinguish a fire before a segment is burnt out, the landscape is considered saved: a partly burnt forest area can regenerate; partially damaged houses can be repaired.

The current wind conditions are displayed in the top right-hand section of the screen (see Figure 1). Wind strength is indicated by the diameter of the circle-the larger the diameter, the stronger the wind. Wind direction is indicated by the direction of the line from the center of the circle-the line points in the direction the wind is blowing. Notice that the fires on the screen (see Figure 1) are burning more intensely and therefore spreading more quickly in this direction. In the top right hand corner of

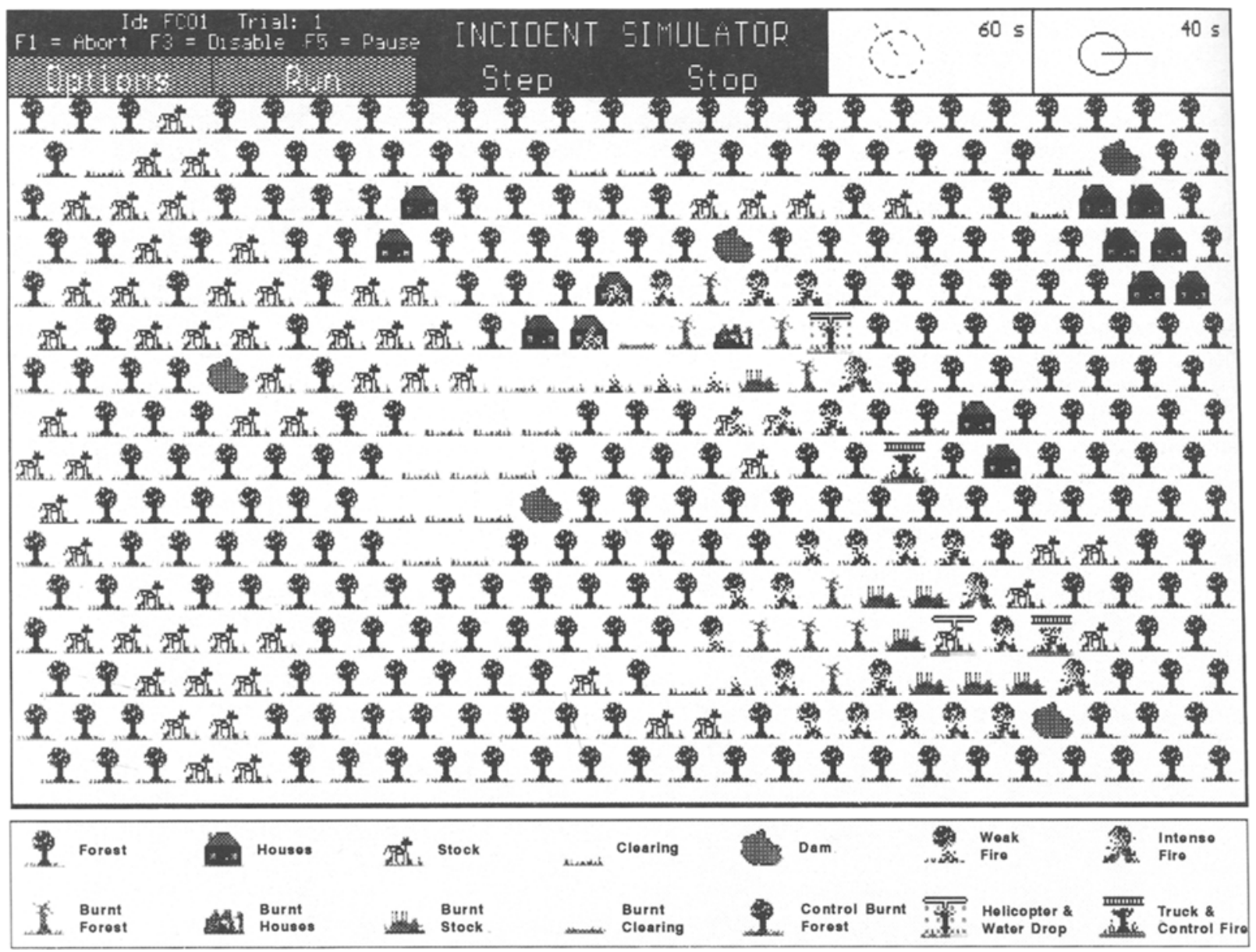




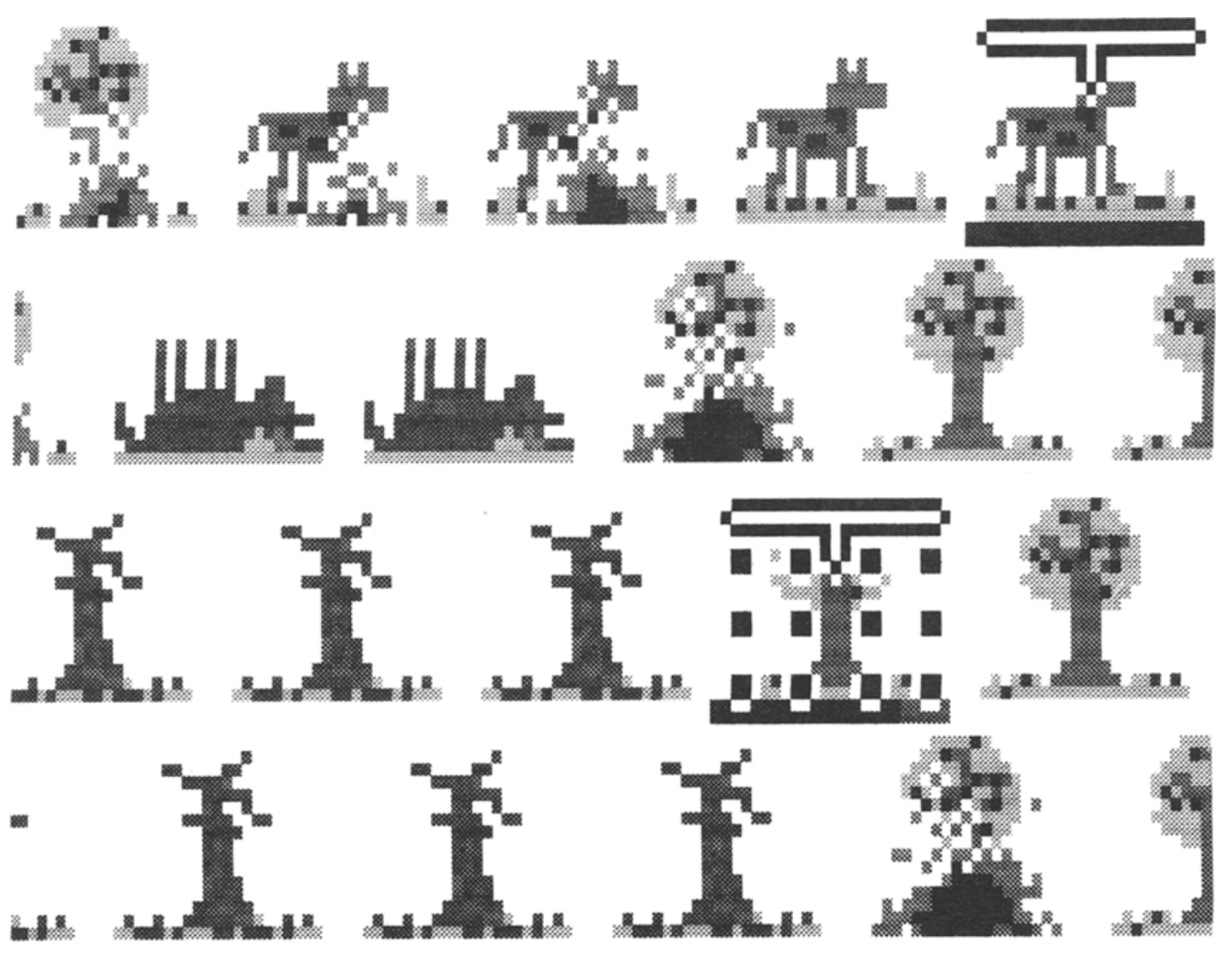

Figure 2. Section of Fire Chief incident simulator screen with trial in progress.

the box displaying these current wind conditions is the current time in the simulation trial.

Wind conditions may change during a trial. You will be given forecasts of these changes in the box on the left of the box displaying the current wind conditions. The forecast wind conditions are indicated by a dotted circle and line display. The time in the simulation trial when these changes are forecast to occur is displayed in the top right-hand corner of this wind forecast box.

Besides fires which are already burning when the trial starts, new fires may break out during the trial. Some of these fires will already be fully established mature fires by the time you receive on-screen notification, whereas others will be newly ignited spot fires which will slowly develop into mature fires before spreading.

As Chief Fire Officer you control the spread of the fires by issuing commands to the crews of the firefighting appliances at your disposal. These appliances are tankers, represented by a ladder symbol; and helicopters, represented by a rotor symbol (see Figure 1). The blue bar below each appliance indicates the amount of water which is currently present in the appliance's tank. You have direct radio contact with the crews manning these appliances. Trucks and helicopters can be sent to any landscape segment and commanded to drop water, to establish a control fire, or to randomly patrol within a designated area, automatically putting out any fires it might find in immediately adjacent landscape segments. If an appliance with an empty, or partially empty, tank arrives at a dam, it will automatically fill with water (unless the dam has already been emptied of water).

You issue these commands to the appliances by a few easy to remember and easy to use mouse and keyboard procedures.

For the simulation trials you will run today, the program has been set for the following conditions:

-Fires spread at different rates and with different intensities through the different types of landscape: traveling most quickly through pastures and burning most intensely in forests.

-Helicopters move faster and drop more water than trucks.

- Some fires may be too intense for an appliance to extinguish. An appliance will give an alarm if a fire cannot be extinguished.

- Only trucks can be commanded to establish a control fire, as this procedure requires firefighting crews to continually bulldoze or clear areas of land with rakes/hoes etc. and to continually mop up any unwanted spot fires which might occur.

-If an appliance is moved from a control fire before the control fire is completed, the unattended control fire will develop and spread as a normal fire.

-Control fires cannot be successfully established in residential areas and any attempt to do so will result in a fully developed house fire.

-If an area completely burns out while occupied by a truck, the truck will also be destroyed. You will be given 
a replacement truck at the location occupied by the destroyed truck at the beginning of that simulation trial.

-You will be given a performance score at the end of each trial, points being deducted for every landscape segment and truck which is destroyed. Forest areas are valued at 2 points, stock pasture at 3 points, clearings at 1 point, houses at 15 points, and trucks at 20 points.

In running all Fire Chief trials, it is important to remember that your aim is to save the landscape and trucks from destruction by fire so as to get as high a score as possible.

\section{Overall Structure of the Fire Chief Program}

The Fire Chief program consists of nine separate modules, which are accessed and controlled via an on-screen menu system (see Figure 3). This figure shows (1) how access to each of the program modules is obtained, (2) how the presentation of trials is controlled, and (3) the file loading and saving options that are available for the contents of each module. Program modules can be classified into three functional types: simulation modules, which simulate the development of fires; control modules, which allow the user to control the functioning of the simulation modules; and report modules, which produce report data on individual trials executed in any of the three simulation modules. A brief overview of Fire Chief functions is provided below. A more detailed description of the functioning of each of the nine individual program modules is available in the Fire Chief User Manual (Omodei \& Wearing, 1993b).

\section{Simulation Modules}

There are three simulation modules, each simulating the development of fires in precisely the same manner. The main functional difference among the three modules is the source of the firefighting commands (decisions) that are issued to the appliances. Commands that can be issued to firefighting appliances comprise commands to move to a new location, to drop water at the current location, to establish a control fire at the current location, and to randomly patrol within a specified landscape area.

Incident simulator. Commands are obtained from a person operating the computer keyboard and mouse. Alternative modes are available to control the timing of trials: either real-time execution (run mode), in which the simulated fires are updated continuously, or commanddriven execution (step mode), in which the simulated fires are updated by a specified amount each time a

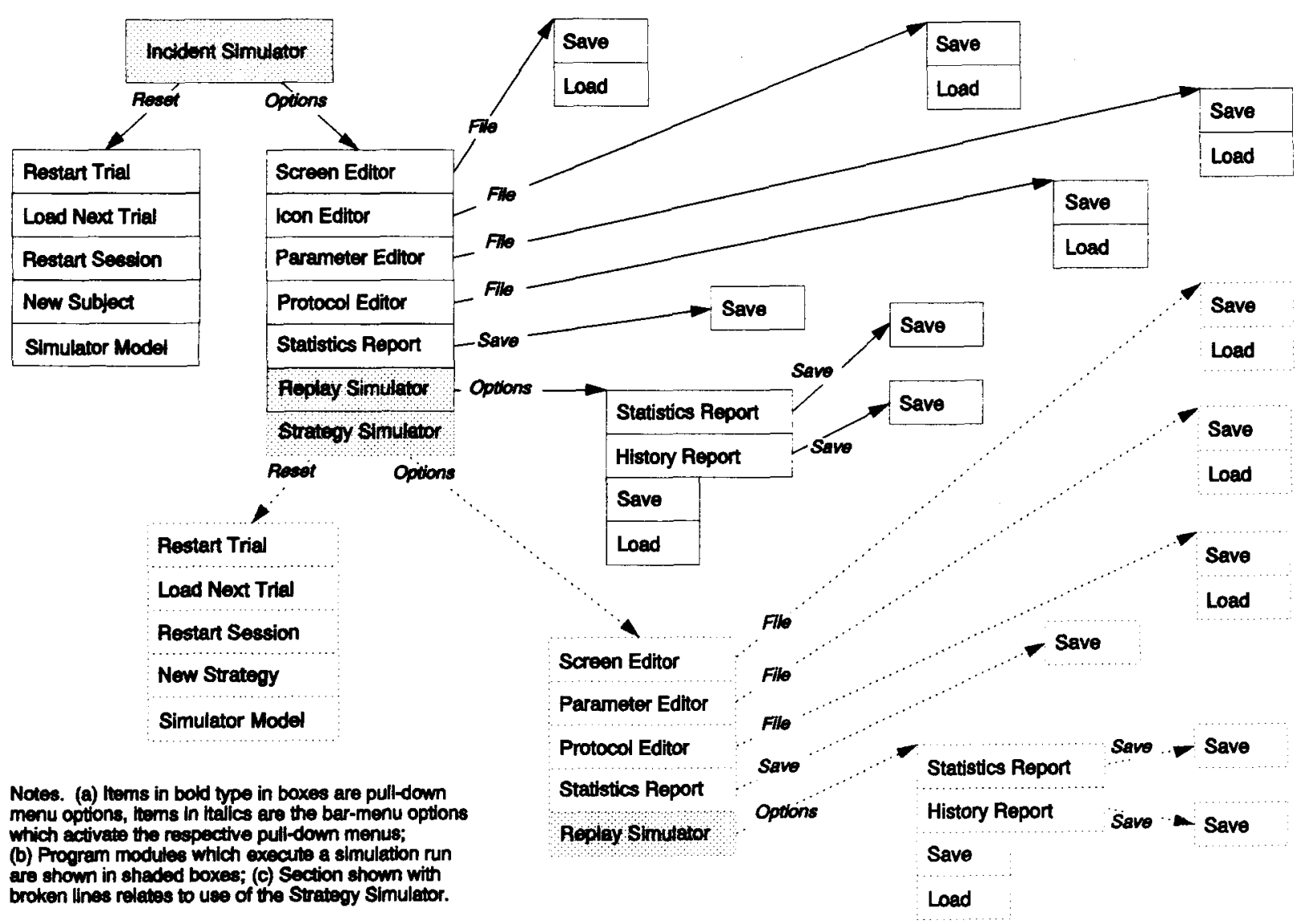

Figure 3. Fire Chief menu system. 
command is entered, with no further fire development occurring until the next command is entered.

Strategy simulator. Commands are generated by special code routines. Program users are provided with the facility to interface their own code routines (written in Turbo Pascal) with the Fire Chief program. These routines can be written to artificially generate commands to control the simulated incidents (e.g., fires). This allows researchers to program theoretically derived decisionmaking strategies (i.e., to program artificial "subjects") and to compare the microstructure of the performance of these strategies with that of human subjects.

Replay simulator. Commands are read from a record of previously entered commands (a command history) allowing for a complete real-time replay of the original trial-akin to the video replays widely used in sporting events. The command history is stored in the form of a command list, with identically structured command lists being generated by persons in the incident simulator and by programmed strategy routines in the strategy simulator. While a trial is being replayed, simple keyboard and mouse operations allow both the experimenter and the subject to pause and to resume replay execution as desired. This dual replay-control facility provides subjects and experimenters with the opportunity to review and analyze prior simulation trials in such a manner as to allow subjects to provide accurate and detailed reports on relevant psychological states (e.g., as verbal protocols).

\section{Control Modules}

Four modules allow the user to customize the appearance and behavior of trials in the simulation modules. The first three modules listed below allow the user to customize the appearance and behavior of each individual simulation trial, whereas the remaining module allows the user to automate the administration and behavior of a series of simulation trials.

Screen editor. This module is used to create different configurations of icons over the screen as a whole. Selecting from a menu of icons corresponding to the various landscape segments (e.g., forest, pasture, clearings, houses, and dams), the user can place segments of any type in any desired position on the screen. The screen editor can thus be used to create different scenarios such as towns, lakes, or forests. In addition to placing landscape segments, the screen editor allows the user to place up to 15 appliances at any desired position on this screen.

Icon editor. The icon editor module supplements the screen editor module by allowing the user to custom design, pixel by pixel, the internal appearance of the icons used to define the landscape scenario, the fires, and the firefighting appliances. For example, a forest icon in the shape of a pine tree could be drawn if one was interested in simulating fires in a pine plantation.

Parameter editor. Direct user access is provided to 45 separate parameters, together with the facility to precisely specify an unlimited number of wind change and fire initiation events throughout a trial. Parameter settings include (1) the particular screen file and icon file to be read into the program at the start of the trial, (2) the number, location, and extent of fires present at the beginning of the trial, (3) how fast the fires spread, (4) how fast the appliances move and how long they take to perform different firefighting functions, (5) the flammability characteristics and the relative value of the different types of landscape segments and fire trucks, (6) when and where additional fires start during a trial, (7) when and how wind conditions change throughout the trial, and $(8)$ the firefighting commands that are available. Fortunately, in most research/training contexts, only a few of these parameters would need to be altered across a series of simulation trials. Default parameter values have been hardwired into the program to provide a set of realistic parameters that can be used as the starting point for creating alternative parameter sets.

Protocol editor. This module provides access to 32 separate protocol settings with which the user can customize the manner in which the Fire Chief program sequentially administers and controls the functioning of a series of simulation trials. Protocol settings include the particular parameter file the program is to load in for each trial, whether the various report files are to be automatically saved at the end of each trial, and the method of allocation of subject identification and trial number codes. By setting up an appropriate set of protocol values, a researcher can, for example, set up a series of trials to implement a particular experimental design before a subject arrives. These trials can then be administered to the subject in rapid succession without the experimenter's having to interrupt the subject either to select trials or to save data. Default protocol values have been hardwired into the program to provide a set of realistic protocols that can be used as the starting point for creating alternative protocol sets.

\section{Report Modules}

Two report modules produce report data on individual trials. These reports may be viewed on screen, printed directly to a printer, or saved in simple text (ASCII) form suitably formatted for subsequent statistical analyses.

Statisties report. This module produces summary statistics concerning the status of the simulated fires and the timing and proportion of the commands issued to the appliances.

History report. This module produces a detailed description of all the commands entered and all the events that have occurred, listed in the order (by time) of occurrence.

\section{The Simulation Model}

In this section we present a brief summary of the methods by which the Fire Chief program models any particular microworld. As indicated above, for ease and consistency in exposition we do so by referring to a forest firefighting microworld. In providing an outline of the simulation procedures used in Fire Chief, we will focus on the procedures by which the real-time execu- 
tion mode (run mode) is implemented. The procedures by which command-driven execution (step mode) is implemented, however, are almost identical. A more comprehensive treatment of the simulation procedures used in the Fire Chief program is available in the Fire Chief User Manual (Omodei \& Wearing, 1993b).

\section{Main Fire Chief Data Structures}

The major data structures implementing Fire Chiefgenerated microworlds comprise the landscape array, the fire list, and the appliance list. These three data structures and their interrelationships are summarized in Figure 4.

Landscape information is stored in a rectangular array (i.e., in a two-dimensional array). Information on fires and information on appliances are stored in linked lists. Information on fires and appliances can be obtained directly from the relevant fields of the elements making up the landscape array. Attention is drawn to the fact that although landscape information is stored in a rectangular array, the environment is modeled as a hexagonal rather than as a rectangular array of uniformly sized segments (e.g., see Figure 2). This allows any one segment to be surrounded by segments that are all equidistant. The hexagonal on-screen appearance is achieved by offsetting the placement of icons in odd-numbered rows to the right of the screen by half the width of an icon. Calculations are performed, wherever necessary, to take into account the on-screen offset of alternating rows. That is, all directions for fire and appliance movements are calculated as if the hexagonal layout is the true landscape layout. For example, fires spread equally to the six bordering screen segments represented by the hexagonal pattern.

\section{Time Control Method Adopted for the Simulation of a Continuous System}

For computational efficiency, discrete processes are used to model the continuous system implied by a realtime microworld. Time increments of a sufficiently small value permit an acceptable approximation of continuous system behavior.

The practical outcome of these discrete modeling processes is that any particular microworld implemented by the Fire Chief program works in artificial time units, referred to as "generations" in Fire Chief. The amount of actual clock time corresponding to each generation is constant from one generation to the next throughout any one simulation trial. This generation time is a usermodifiable parameter (the default value is $0.2 \mathrm{sec}$ ). The amount of time in the simulated microworld which corresponds to each such generation can be arbitrarily defined. Consequently, scenarios that usually take many

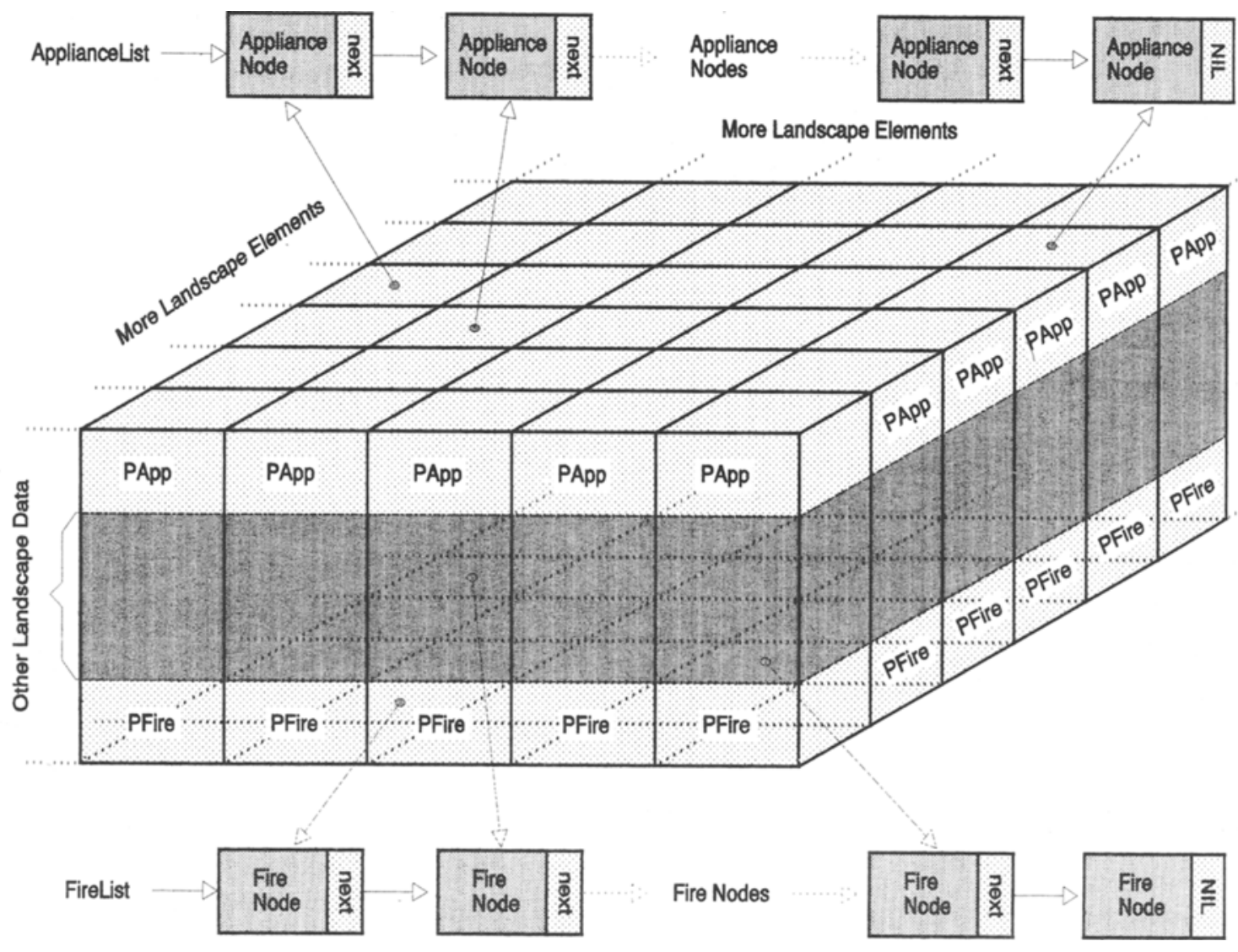

Figure 4. Interrelationships among main Fire Chief data structures. 
hours to develop (e.g., a large-scale forest fire) can be simulated in laboratory trials of just a few minutes. Millisecond accuracy is implemented in all Fire Chief timing routines.

The amount of time is a user-modifiable parameter. The value $0.2 \mathrm{sec}$ was chosen for the time the computer allows for each generation because this is sufficiently short to allow fire development to appear smooth and continuous to the subject. Generation times as small as $.001 \mathrm{sec}$ can be selected. However, the CPU speed of the particular computer running the simulation and the processing complexity of the particular scenario being implemented do limit just how short a generation time can be implemented in real time. This is discussed later in the section on hardware/software requirements.

\section{The Model for Implementing Incident Development}

The specific equations used to model incident development in the Fire Chief program are presented in the Appendix. In summary, the procedures used to update fire status in the landscape segment are performed in the following order: (1) the intensity of all fires is updated for current wind and moisture conditions, and for presence of fire in adjacent segments; (2) all fires are evaluated for possible extinction or for degree of transmission threat posed to adjacent unburning landscape segments; and (3) all unburning but threatened landscape segments are evaluated for possible ignition.

The Fire Chief model departs from existing landscapebased simulations in two significant ways. First, the environment is modeled as a hexagonal rather than a rectangular array of uniformly sized segments (as described in detail above). Second, the equations used to assess fire intensity and fire spread were empirically derived by investigating the ability of equations of various forms to generate fires that would spread according to the "tear drop" shape in which actual fires are observed to spread (see Luke \& McArthur, 1978). Similar patterns of incident spread are also observed in pest infestations.

\section{Important Issues in the Development and Use of Computer-Simulated Microworlds: The Fire Chief Program as an Illustrative Case}

In developing and testing the Fire Chief program in experimental settings, we were forced to address several issues that apply to the development and use of computer-simulated microworlds more generally. In the remainder of this paper we introduce the more salient of these issues, illustrating each issue by indicating how it has been specifically addressed in the Fire Chief program. These issues include the following: flexibility and generality with respect to the structure of the microworlds that can be created, adequate psychological validity, automation of experimental procedures (including trial administration, subject debriefing, and data logging), capacity to interface and test computer simulations of psychological processes, and adequate program testing and program documentation.

\section{Flexibility and Generality in the Microwordds That Can Be Created}

Flexibility and generality are important in microworldgenerating programs for several reasons: First, flexibility allows particular microworlds to be developed to address specific research questions, in varied research contexts, with diverse subject populations. Second, it allows any one research group to assess the extent to which the findings obtained are of restricted generality by being either "task bound" or "parameter bound."

Fire Chief in its current form allows for markedly different incidents to be simulated, ranging from minor modifications of the default forest firefighting scenario (a self-generating process) to major modifications of the default scenario to create incidents that possess a very different underlying development history and response to control interventions (e.g., sea rescue, accidental roadtanker spillage, crowd control, etc.). Such program flexibility is achieved by providing the user with control over program behavior at three progressively deeper levels, as follows:

Graphical appearance. A variety of incidents that follow development histories analogous to that which occurs in fire behavior and fire control may be modeled simply by altering the "cover story" and redesigning the graphical icons to support this cover story (e.g., plague infestation and spread and its control with insecticide).

User-modifiable parameter values. Altering the pattern of values of selected parameters, including the enabling/disabling of several parameters, allows for the implementation of markedly different underlying models of incident occurrence, development, and control. For example, by disabling parameters used in the simulation of self-generating processes (such as landscape flammability characteristics) and by setting the incident decay parameter to a suitable value, one can model a completely non-self-generating process (such as a chemical tanker spillage or a nuclear power plant explosion). Furthermore, by completely disabling incident spread parameters, one can use Fire Chief to simulate incidents involving the rescue of ships at sea and the detection of hostile aircraft in an air defense system. Moreover, by selectively weighting the values attributed to the various types of segments that make up the microworld environment, one can create scenarios in which the aim is to enhance, rather than retard, the simulated incidents (e.g., agricultural husbandry, market trends, etc.).

Incident development algorithms. Fire Chief's relatively simple programming interface allows researchers to substitute their own code (in Turbo Pascal) in order to specify alternative relationships among the variables that constitute the incident system and its response to potential user actions. For example, by reprogramming the routine that determines the rate of incident development within a segment (Appendix, Equation A1), the user can introduce probabilistic factors, as would be the case if landscape vegetation characteristics were variable. More radical alteration of these routines allows the user to specify completely different patterns of incident devel- 
opment and spread, such as the behavior of commercial markets.

\section{Psychological Validity: How Closely the Microworld Reflects Psychological Reality}

To design a microworld that adequately captures reality for the investigation of psychological processes, it is necessary to define precisely what characteristics of the external reality need to be captured. The microworld must possess "psychological validity" as distinct from "physical validity." Psychological validity requires that the simulated microworld engage the same psychological processes in the subject that would be engaged in the external reality being modeled. With respect to the psychological study of complex decision-making behaviors, therefore, the microworld must present to the subject a decision-making environment that is adequately complex, dynamic, and time bound. A more extensive discussion of these three characteristics has already been presented in the introductory section to this paper. Furthermore, for a comprehensive study of the factors associated with such decision making, the microworld must be such that the motivational, intentional, and affective processes that are typically elicited in such situations are also elicited by the microworld (Omodei, 1993; Omodei \& Wearing, 1991).

Two aspects of computer-simulated microworlds which are fundamental to the implementation of such psychologically valid decision-making environments are the "cover story" that is supplied and the adoption of a nonintrusive interface. Each of these aspects of simulation use are described below.

Adoption of an adequate cover story. The preceding discussion of psychological validity emphasizes the importance of presenting subjects with an appropriate "cover story." As indicated in an earlier section of this paper, any specific microworld can be implemented by appropriate customization of Fire Chief screen layout and parameter settings. In describing the particular microworld to subjects, an appropriate "cover story" must be presented whereby real-world meanings are ascribed to the various program data structures and parameters. These meanings are graphically illustrated and supported by the particular icons which are designed for use in conjunction with the cover story.

Specific cover story selection has important implications for the psychological validity of any findings obtained with its use. Subjects bring to any task their own implicit understandings of the reality implied by the particular cover story supplied. Unless the underlying structure of the task (specified by the underlying mathematical model) is congruent with subjects' preexisting understandings, it will not be possible to validly interpret subjects' decision behavior and overall task performance. Brehmer, Leplat, and Rasmussen (1991) have provided a more extensive discussion of the need to make sure that simulations do not contradict any laws of nature of which subjects might potentially be aware. It is particularly important that hidden aspects of system behavior correspond to subjects' everyday experience of the realworld situation being simulated. This requires that the microworld embody at least those physical aspects of the external reality of which a subject can be expected to be aware. That is, psychological validity requires that the task reproduce the functional behavior of the real system at least at the level observable by a human participant. For example, in simulating forest fires, psychological validity requires that the simulation embody only the principles of fire chemistry and fire physics of which the violation would be obvious to potential users of the simulation task. Conversely, it is equally important that the provided cover stories do not suggest to subjects functional relationships that are not actually implemented in the simulated microworld scenario.

A related validity concern is the researchers' assumption that subjects will in fact "accept" the cover story, particularly with regard to the performance goals that are set for the subjects (e.g., save as much forest as possible from fire). This need to pay attention to the acceptability to subjects of any particular cover story is illustrated by our experiences with a previous microworld simulation program we had developed. This microworld, Decoy (Omodei, 1990), which relies on perceptual-motor skills, is somewhat similar in structure to Mané and Donchin's (1989) Space Fortress game. Decoy requires subjects to imagine that they are in an armored vehicle in an enemy-occupied mine field and are responsible for destroying enemy attack craft while avoiding these mines. Several subjects in pilot investigations with this microworld objected to the aggressive, militaristic overtones implied by the cover story and therefore either did not try very hard or tried not to damage the attack craft.

Use of the default forest firefighting microworld illustrates several validity-related issues. First, subjects are likely to accept fire control as a nontrivial and valued activity. Furthermore, subjects are likely to have some general familiarity with the processes of fire development and fire control, based on mass media reporting. Such general familiarity reduces the amount of time required to familiarize subjects with the program and reduces the possibility of subjects bringing to the task varying and erroneous expectations concerning simulation behavior.

Although initial program development was somewhat focused on establishing psychological validity with respect to forest firefighting alone, we argue that forest firefighting is a prototypical example of a range of dynamic control situations that involve spatiotemporal decisionmaking processes (cf. Brehmer \& Løvborg, 1991). Firefighting is frequently used to illustrate the sorts of decisionmaking demands that many complex dynamic systems place on persons who attempt to control them. For example, common language includes many instances in which firefighting terms are used as metaphors to describe reallife complex situations: "tackling the problem on all fronts"; taking chances with the hope of "putting out any fires" that might occur as the result of oversights. This suggests that the structure of a firefighting situation is isomorphic with many situations in which action 
must be taken in continually changing and uncertain environments.

An additional justification for focusing on firefighting in the initial stages of program development is that fire development and fire control allow for wide variety in the cognitive complexity of the task presented to subjects, ranging from quite simple scenarios to very complex ones. A simple scenario for a forest fire microworld, for example, could be one in which the fire chief is required to command a single appliance to drop water on a slowly moving fire in a simple forest landscape with stable wind conditions. A complex forest fire scenario, on the other hand, could be one in which the fire chief is required to anticipate fire behavior in differing terrain and wind conditions; to control several helicopters and trucks that move at different speeds and drop water at different rates; to issue commands to appliances to drop water, to establish a control fire, or to automatically patrol; and to prevent fire trucks from being destroyed. The outcome, therefore, of focusing initially on firefighting was a program shell that is capable of generating microworlds of a wide range of complexities.

Nevertheless, the extensive editing facilities of the Fire Chief program allow for many different psychologically valid realities to be presented to potential research subjects.

Adoption of a nonintrusive interface design. The human-computer interface represents the bridge between the subject and the microworld that is being simulated. Characteristics of this interface determine not only how a simulated microworld is presented to the subject but also (and perhaps even more importantly) how the subject can act in this world. It is important, therefore, that the interface be sufficiently sophisticated to encompass the dynamic complexity of the microworld, while also being easy to interpret and easy to use. If subjects are to experience a computer-administered task as a psychologically valid microworld, all their experiences should derive from their assigned "role" and their rolerelated decision making in that microworld, and not from any specific characteristics of the interface itself. For example, the simulation interface should be designed so that subjects' attention need not be removed from the simulated scenario itself to the relatively artificial and mechanical aspects of the interface. Particular care was therefore taken with Fire Chief to implement command-entry procedures that are natural, nonintrusive, and quickly routinized-as is the case in video and arcade games.

The extent to which the Fire Chief interface requires subjects to focus attentional resources on the manipulation of the mouse and keyboard was assessed in one of the studies we have conducted with Fire Chief (Omodei $\&$ Wearing, 1989). Subjects were given a set sequence of predetermined commands to input as quickly as they could. The average time to input commands under these conditions was substantially less than was the case when such commands required prior complex decision-making activity $(M=1.29 \mathrm{sec}, S D=0.21 \mathrm{sec}$, vs. $M=2.89 \mathrm{sec}$,
$S D=0.83 \mathrm{sec}$ ). That is, the average time to input decisions was more than twice that required for the mechanical procedures for command entry and there was substantially less variability across subjects in the execution of these procedures. It can be concluded, therefore, that a sufficiently large component of the variance in the time interval between successive decision input can be attributed to the higher level cognitive processing involved in the formulation of decisions.

Computer interfaces as a genuine component of reallife decision contexts. In discussing the issue of psychological validity with respect to the use of a computerbased interface to a simulated microworld, it is perhaps useful to draw attention to the increasing frequency with which remote video terminals themselves have become a part of many real-life decision environments. That is, there are many examples in contemporary decision making in which decision makers remain somewhat removed from the sphere of action, typically operating in front of a computer screen and relying on remote data-sensing devices for relevant information. For example, computerbased systems are now used in the strategic allocation of vehicles and personnel in the operation of emergency services (e.g., police, fire, and ambulance services), in the robotic control of industrial processing plants, and in the cockpit of modern airplanes. This development has several implications for the creation of psychologically valid experimental tasks. Not only does it suggest a greater variety in the sorts of cover stories that can be created, it also increases the likelihood that such cover stories, and associated interfaces, will be accepted as "valid" by potential subjects.

\section{Automatic Implementation and Administration of Complex Experimental Designs}

The complexity of microworld scenarios, and therefore of the psychological processes that can be elicited by them, raises the possibility of (and often necessity for) quite complex experimental designs. Such complexity creates the potential for costly errors to be made by the experimenters who supervise the administration of the relevant experimental trials. As indicated above in summarizing the Fire Chief program's control modules, facilities are provided that allow the user to fully customize and automate the sequencing and the behavior of a series of simulation trials, including the allocation of identification codes and the saving of report files.

\section{Nonreactive Subject Debriefing Techniques}

The complexity of the demands that simulated microworlds place on subjects, together with the validity requirement that subjects immerse themselves in the artificial world, precludes the use of most direct techniques for obtaining access to on-task psychological experiences (e.g., techniques such as thinking aloud and task interruption). What are required are posttask debriefing procedures that are sufficiently sensitive to make salient for subject recall the relevant on-task experiences. This implies the need for a flexible playback facility that can re- 
capture not only the content but also the timing of a subject's interactions with the microworld. Consequently, the Fire Chief replay simulator module includes various pause, command by command stepping, and fast forward options, which allow the researcher to utilize a variety of debriefing procedures. A refinement that appears to be unique to Fire Chief as a microworld-generating program is the implementation of a dual replay control facility. This dual control facility allows for the application of a variety of sensitive interviewing techniques, such as those associated with event-stimulated recall procedures (cf. Meichenbaum \& Butler, 1979).

\section{Extensive Data Logging}

In interacting with complex microworlds, subjects typically generate large amounts of data that can be interpreted only when examined in conjunction with current events in the simulated world. Consequently, as trials are run, both summary performance data and sequential command and event histories must be automatically generated. As indicated above in summarizing the Fire Chief program's report modules, these data may be viewed on screen, printed directly to a printer, or saved in simple text (ASCII) format suitably formatted for subsequent statistical analyses.

\section{Facility to Test Computer Models of Complex \\ Human Processes: Interfacing Artificial Subjects}

Computer simulation of theoretically predicted psychological processes is emerging as a powerful technique in theory development and testing (cf. Boden, 1988; Reichert \& Dörner, 1988; Schaub, 1990). Particular care has therefore been taken in the design and documentation of the Fire Chief program to allow users to program and directly interface code that artificially generates commands to the simulated microworld appliances. This facility allows, for example, for the modeling of cognitive strategies for managing the microworld task environment.

\section{Adequate Program Testing}

As with any complex computer program, reliability and validity require that the software development cycle include testing of the program in use. We have successfully completed a series of experimental studies addressing a range of psychological issues concerning motivational, emotional, behavioral, and cognitive factors (see Omodei, 1993; Omodei, Olver, Gilbert, \& Wearing, 1993; Omodei \& Wearing, 1989, 1991, 1992, 1994). The task has been found to allow for sensitive discriminations to be made in performance and subjective experience across a range of task conditions and person characteristics. The studies conducted so far have addressed a diverse set of research questions; included a large number of variables; adopted a range of methodological techniques, both qualitative and quantitative; and implemented several complex experimental designs.

\section{Adequate Documentation: The Need for a Comprehensive Manual}

Full, accurate, and efficient use of any complex simulation program requires adequate program documentation. This is especially the case for a program that possesses the features outlined in the immediately preceding sections of this paper. Consequently, a detailed user manual has been written to accompany the Fire Chief program. The Fire Chief User Manual (Omodei \& Wearing, 1993b) provides accurate, comprehensive, and easily accessible information concerning program use. The manual is extensively illustrated with sample screen output and includes specific suggestions and techniques for program use in a variety of research and training contexts. These include detailed suggestions and instructions for the design and conduct of experimental investigations into various psychological processes and a programming guide for coding and testing artificial decision-making strategies.

\section{Hardware/Software Requirements}

The minimum hardware for running Fire Chief comprises an 80286-based personal computer (IBM AT compatible) with a math coprocessor, a Microsoft-compatible mouse, and an EGA-compatible graphics card and monitor. To run complex simulation trials in real time, a minimum of an 80386-based machine with a math coprocessor is recommended. An 80286-based computer running at a clock speed of $12 \mathrm{MHz}$ with an 80287 math coprocessor requires up to $0.200 \mathrm{sec}$ to process a generation if there are many simulated landscape segments on fire.

To run Fire Chief, the only software requirements are the MS-DOS operating system, a Microsoft-compatible mouse driver, and the Fire Chief program file, FCHIEF. EXE, which is approximately $300 \mathrm{~K}$ in size (compiled from approximately 15,000 lines of source code).

Availability. The Fire Chief program and accompanying Fire Chief User Manual are available to interested researchers. As indicated in preceding sections of this paper, Fire Chief has been developed as an open software environment (Turbo Pascal) allowing the program user to interface code to specify (1) alternative relationships among the variables that constitute the incident system and its response to potential user commands and (2) artificially generated commands.

The Fire Chief program therefore comprises the executable code (FCHIEF.EXE) together with the necessary precompiled units and source code units to implement the open software environment outlined above. We ask for a check for $\$ 100$ US or $\$ 140$ Australian to cover costs of materials, postage, and handling. This check should be made out to the Department of Psychology, University of Melbourne, and should be sent to Alexander Wearing, Department of Psychology, University of Melbourne, Parkville, Victoria, 3052, Australia. Requests for technical information on the Fire Chief program should be addressed to Mary Omodei, School of Be- 
havioural Health Sciences, La Trobe University, Bundoora, Victoria, 3083, Australia; or by electronic mail to m.omodei@latrobe.edu.au.

\section{REFERENCES}

Ackerman, P. L. (1987). Individual differences in skill learning: An integration of psychometric and information processing perspectives. Psychological Bulletin, 102, 3-27.

Boden, M. (1988). Computer models of mind: Computational approaches in theoretical psychology. New York: Cambridge University Press.

BREHMER, B. (1992, fall). Research in Uppsala continues to focus on problems of dynamic decision making. Brunswik Society Newsletter, $7,1-2$.

Brehmer, B., \& Allard, R. (1991). Dynamic decision making: The effects of task complexity and feedback delay. In J. Rasmussen, B. Brehmer, \& J. Leplat (Eds.), Distributed decision making: Cognitive models for cooperative work (pp. 319-355). New York: Wiley.

BREHMER, B., \& DöRNER, D. (1993). Experiments with computersimulated microworlds: Escaping both the narrow straits of the laboratory and the deep blue sea of the field study. Computers in Human Behavior, 9, 171-184.

BREHMER, B., LEPLAT, J., \& RASMUSSEN, J. (1991). Use of simulation in the study of complex decision making. In J. Rasmussen, B. Brehmer, \& J. Leplat (Eds.), Distributed decision making: Cognitive models for cooperative work (pp. 373-386). New York: Wiley.

BREHMER, B., \& LøVBORG, L. (1991). NewFire-A flexible system for running simulated fire-fighting experiments. In Proceedings of the Second Mohawc Workshop: Cognitive processes and resources, Manchester, 27-28 November, 1990 (pp. 187-200). Roskilde: Risø National Laboratory.

Donchin, E. (1989). The Learning Strategies project. Introductory remarks. Acta Psychologica, 71, 17-22.

Dörner, D., KREUZig, H. W., Reither, F., \& StäUdel, T. (1983). Lohhausen: Vom Umgang mit Unbestimmtheit und Komplexität [Lohhausen: On dealing with uncertainty and complexity]. Bern: Huber.

Dörner, D., STäUdel, T., \& Strohschneider, S. (1988). Moro: Programmdokumentation [Moro: Program Manual] (Memorandum XX). Bamberg, Germany: University of Bamberg, Lehrstuhl Psychologie II.

KANFER, R., \& ACKERMAN, P. L. (1989a). Dynamics of skill acquisition: Building a bridge between intelligence and motivation. In R. J. Sternberg (Ed.), Advances in the psychology of human intelligence (Vol. 5, pp. 83-134). Hillsdale, NJ: Erlbaum.

KANFER, R., \& ACKERMAN, P. L. (1989b). Motivation and cognitive abilities: An integrative/aptitude-treatment interaction approach to skill acquisition. Journal of Applied Psychology, 74, 657-690.

LuKE, R. H., \& MCARTHUR, A. G. (1978). Bushfires in Australia. Canberra: Australian Government Publishing Service.

ManÉ, A., \& Donchin, E. (1989). The Space Fortress game. Acta Psychologica, 71, 17-22.

Meichenbaum, D., \& Butler, L. (1979). Cognitive ethology: Assessing the streams of cognition and emotion. In K. Blankstein, P. Pliner, \& J. Polivy (Eds.), Advances in the study of communication and affect: Assessment and modification of emotional behavior (Vol. 6, pp. 306-321). New York: Guilford.

MORRIS, N. M., \& RouSE, W. B. (1985). The effects of type of knowledge upon human problem solving in a process control task. IEEE Transactions on Systems, Man, and Cybernetics, SMC-15, 698-707.

OMODEI, M. M. (1990). Decoy : A dynamic decision making task [Computer program]. University of Melbourne, Department of Psychology.

OMODEI, M. M. (1993). Fire Chief: A computer simulated real time dynamic decision making task. Unpublished doctoral dissertation, University of Melbourne.

Omodei, M. M., Olver, N. D., Gilbert, J., \& Wearing, A. J. (1993, August). Controlling real time dynamic environments: Relevant task and person characteristics. Paper presented at the Research Conference on Subjective Probability, Utility, and Decision Making, Aix-En-Provence, France.

OMODEI, M. M., \& WeARING, A. J. (1989, November). Cognition and affect in dynamic decision making: A study of involvement, boredom, and anxiety in a forest firefighting simulation. Paper presented at the meeting of the Psychonomic Society, Atlanta.

Omodei, M. M., \& Wearing, A. J. (1991, August). Decision making in complex dynamic settings: A theoretical model incorporating motivation, intention, affect, and cognitive performance. Paper presented at the Research Conference on Subjective Probability, Utility, and Decision Making, Fribourg.

OmodeI, M. M., \& WeARING, A. J. (1992, September). Research applications of the Fire Chief microworld simulation program. Paper presented at the Conference of the Australian Psychological Society, Armidale.

OMOdEI, M. M., \& WeARING, A. J. (1993a). Fire Chief (Version 2.2) [Computer program]. University of Melbourne, Department of Psychology.

Omodei, M. M., \& Wearing, A. J. (1993b). Fire Chief user manual (Version 2.2). University of Melbourne, Department of Psychology (ISBN: 086744028 7).

Omodei, M. M., \& WeARING, A. J. (1994). Perceived difficulty and motivated cognitive effort in a computer-simulated forest firefighting task. Perceptual \& Motor Skills, 79, 115-127.

REICHERT, U., \& DöRNER, D. (1988). Heurismen beim Umgang mit einem "einfachen" dynamischen System [Heuristics in the control of a "simple" dynamic system]. Sprache \& Kognition, 7, 12-24.

SchaUb, H. (1990). The year of the gardener-behavior in a complex situation. In Proceedings of the Second Mohawc Workshop: Cognitive Processes and Resources, Manchester, 27-28 November, 1990 (pp. 73-90). Roskilde: Risø National Laboratory.

Schneider, W., Dumais, S. T., \& Shiffrin, R. M. (1984). Automatic and control processing and attention. In R. Parasuraman \& D. R. Davies (Eds.), Varieties of attention (pp. 1-27). New York: Academic Press.

SchNeIDER, W., \& SHIFFrin, R. M. (1977). Controlled and automatic human information processing: I. Detection, search, and attention. Psychological Review, 84, 1-66.

Shiffrin, R. M., \& Schneider, W. (1977). Controlled and automatic human information processing: II. Perceptual learning, automatic attending, and a general theory. Psychological Review, 84, 127-190.

SPRINSOCK, E. (1985). Fire [Computer program]. San Francisco: Freeware.

TURKLE, S. (1984). The second self: Computers and the human spirit. London: Granada.

\section{APPENDIX \\ The Specific Equations Used to Model Incident Development in the Fire Chief Program}

Fire intensity in a landscape segment. Equation $\mathrm{A} 1$ assesses the current fire intensity in a landscape segment, taking into account the wind conditions, vegetation characteristics, and whether adjacent segments are burning (the Headfire Adjustment Factor).

$$
F_{i j}=D_{i j} \times B_{i j} \times X_{i j} \times R_{a}\left(.4+e^{\left(W_{i j}+H_{i j}\right) I n S}\right)-M_{i j} \text {, }
$$

where $F_{i j}=$ fire intensity in landscape segment $i j ; D_{i j}=$ density of combustible fuel in landscape segment $i j ; B_{i j}=$ build-up factor for fire in landscape segment $i j ; X_{i j}=$ process extinction level for fire in landscape segment $i j ; \mathbf{R}_{a}=$ fire spread rate in landscape segment of type $a ; W_{i j}=$ wind direction factor for landscape segment $i j ; H_{i j}=$ headfire adjustment factor for landscape segment $i j ; S=$ wind strength; and $M_{i j}=$ water moisture content in landscape segment $i j$.

The density of combustible fuel $\left(D_{i j}\right)$ is set to the amount of combustible fuel $\left(C_{i j}\right)$ present at the time of ignition of the fire in the particular landscape segment. If the fire is extinguished before it consumes all the combustible fuel in the landscape segment, and if the segment is then reignited at some later time, then $D_{i j}$ is reevaluated for this remaining amount of fuel. 
The build-up factor $\left(B_{i j}\right)$ indicates how established the fire in the particular landscape segment is. This factor will be set to 1.0 whenever a fire is ignited from a fire in an adjacent segment. If, however, the fire is newly ignited in the particular segment, the value of the build-up factor is set to 0.0 and is gradually increased to 1.0 over the following $6 \mathrm{sec}$, following an approximately exponential development function.

The process extinction factor $\left(X_{i j}\right)$ specifies the extent to which an incident has decayed since initial ignition. In simulating fire development, the process extinction factor will always take on the value 1.0 as fire development can be regarded to be a fully self-generating process.

The wind direction factor $\left(W_{i j}\right)$ represents the extent to which a fire is/will burn in the current wind direction. This wind direction factor is calculated at the time of ignition and is determined by the relationship between the direction from which a fire was/is ignited and the current wind direction: ignited directly into wind, $W_{i j}=3$; ignited diagonally into wind, $W_{i j}=2$; ignited diagonally against wind, $W_{i j}=1$; and ignited directly against wind, $W_{i j}=0$.

The headfire adjustment factor $\left(H_{i j}\right)$ represents the extent to which fire intensity is increased due to the presence of fires in adjacent segments. The headfire adjustment factor for segment $i j$ is set to 0.5 if the following conditions are met at time of ignition of segment $i j$ : (1) the wind direction factor is/was 2 , and (2) the adjacent segment in the current wind direction is/was on fire. Otherwise, the headfire adjustment factor is set to 0.0 . These values for the headfire adjustment factor model the effects of preheating which are observed in naturally occurring fires and therefore results in a more realistic fire spread shape.

Unburnt fuel in a landscape segment. Equation A2 uses the fire intensity value from Equation A1 to estimate the amount of combustible fuel consumed in the segment during the simulated time unit and thus the amount of combustible fuel that remains.

$$
C_{i j}(t)=C_{i j}(t-1)-F_{i j} \times g,
$$

where $C_{i j}(t)=$ combustible fuel in landscape segment $i j$ at time $t ; C_{i j}(t-1)=$ combustible fuel in landscape segment $i j$ at time $t-1 ; F_{i j}=$ fire intensity in landscape segment $i j$; and $g=$ length of a simulation time unit.

Fire threat from a burning segment to an adjacent segment. Equation A3 evaluates the potential fire intensity in an as yet unburning segment should it be ignited from an adjacent burning segment. The potential fire intensity value thus calculated is taken to represent the degree of threat that the burning segment poses to that particular adjacent segment.

$$
T_{i j \rightarrow x y}=W_{i j \rightarrow x y} \times F_{i j} \times X_{x y},
$$

where $T_{i j \rightarrow x y}=$ threat from landscape segment $i j$ to landscape segment $x y ; W_{i j \rightarrow x y}=$ wind direction factor from segment $i j$ to segment $x y ; F_{i j} \stackrel{\rightarrow}{=}$ fire intensity in landscape segment $i j$; and $X_{x y}=$ process extinction level for fire in landscape segment $x y$.

If an unburnt landscape segment is adjacent to more than one burning segment and each of these adjacent segments burns out in the same simulation update cycle, the unburnt segment is considered to be ignited from that segment which poses the greatest threat $\left(T_{i j \rightarrow x y}\right)$.

Moisture evaporation factor. Equation A4 calculates the factor by which the moisture level in all landscape segments is to be reduced during each simulation update cycle. The effect of the value of $E$ thus calculated is to reduce the amount of water in any particular landscape segment by $50 \%$ every $10 \mathrm{sec}$.

$$
E=e^{(\operatorname{In}(0.986) \times g \times L \div 0.200)},
$$

where $E=$ moisture evaporation factor; $g=$ length of a simulation time unit; and $L=$ length of update cycle in seconds.

(Manuscript received November 10, 1993; revision accepted for publication August 1, 1994.) 